\title{
NOMA-Based Overlay Cognitive Satellite-UAV-Terrestrial Networks with Multiple Primary Users
}

\author{
Rui Liu $\mathbb{D}^{1},{ }^{1}$ Kefeng Guo $\mathbb{D}^{1},{ }^{1,2}$ Kang An $\mathbb{D}^{3},{ }^{3}$ Shibing Zhu, ${ }^{1}$ and Haifeng Shuai $\mathbb{D}^{1}$ \\ ${ }^{1}$ School of Space Information, Space Engineering University, Beijing 101 407, China \\ ${ }^{2}$ College of Electronic and Information Engineering, Nanjing University of Aeronautics and Astronautics, Nanjing 210 016, China \\ ${ }^{3}$ Sixty-third Research Institute, National University of Defense Technology, Nanjing 210 007, China
}

Correspondence should be addressed to Kefeng Guo; guokefeng.cool@163.com

Received 7 December 2021; Accepted 19 January 2022; Published 12 February 2022

Academic Editor: Long Yang

Copyright ( 2022 Rui Liu et al. This is an open access article distributed under the Creative Commons Attribution License, which permits unrestricted use, distribution, and reproduction in any medium, provided the original work is properly cited.

\begin{abstract}
Satellite communication is becoming more and more significant in present and future communication systems for its unique advantages of strong survivability and seamless coverage, which can offset the defects of terrestrial communication such as being restricted by terrain and little coverage. Moreover, unmanned aerial vehicle (UAV)-assisted communication is considered as a promising development direction due to its flexibility and expansibility in the integrated satellite-terrestrial networks (ISTN). In addition, to overcome the spectrum shortage and low spectrum utilization problems, cognitive radio and nonorthogonal multiple access (NOMA) has been widely utilized to enhance spectrum efficiency, which is considered as the key technologies of the next generation communication. In this regard, our paper investigates the performance of cognitive satellite-UAV-terrestrial networks with NOMA scheme and multiple primary users. Specifically, the exact expressions of outage probability (OP) and ergodic capacity for both primary network and secondary network are derived. To gain further views, the asymptotic expression of OP and diversity orders for the two networks are provided. Finally, through numerical simulations, the correctness of the theoretical derivations is verified and the influences of critical variables on system indexes are also analyzed.
\end{abstract}

\section{Introduction}

For the next generation wireless communication system, large coverage, high speed, low energy consumption, and high spectrum utilization are considered to be its main characteristics. Satellite communication (SatCom) is considered as an indispensable part, which can help realize the seamless connection of global communication and deal with emergencies in disaster areas or remote areas $[1,2]$. Consequently, integrated satellite-terrestrial network (ISTN) is becoming a hot research direction over recent years, which can realize the complementarity of SatCom and terrestrial wireless communication [3]. Moreover, unmanned aerial vehicle (UAV)-assisted communication has attracted the interests of many experts, which can be rapidly deployed and adjusted in time [4]. Hence, the UAV-assisted ISTN, namely, integrated satellite-UAV-terrestrial network (ISUAVTN) has many practical applications in the situation of the surge of wireless terminals [5]. However, limited spectrum resources will restrict the development of ISUAVTN. In this regard, cognitive radio (CR) and nonorthogonal multiple access (NOMA) are utilized to solve the above problem by enhancing spectral efficiency (SE) [6-8].

1.1. Related Works. As a promising network architecture for the future communications, ISTN has momentous practical significance, which is able to overcome obstacles and geographical constraints [9-11]. In [10], the authors utilized auction mechanism to reduce the system consumption of ISTN, and Hungarian algorithm was applied to solve the optimization problem. An integrated multibeam method was designed to maximize aggregated rate of ISTN in [11]. The authors of [12] discussed the outage performance of an unlink ISTN with co-channel interference (CCI) and inherent hardware impairments (HIs), which utilized partial relay selection scheme to choose the optimal relay. In [13], the distinct adaptive transmission schemes were developed 
to serve ISTN with actual applications. A new dual-hop ISTN with HIs was investigated in [14], where a land mobile satellite (LMS) was regarded as the relay. The authors of [15] investigated the physical layer security of ISTN with multiple relays, users, and eavesdroppers, where opportunistic user relay selection scheme was adopted to enhance secrecy performance. In [16], the best-user and user-fairness scheduling schemes were proposed to enhance the outage performance of ISTN by utilizing the optimal beam-forming (BF) weight vectors of relay.

Meeting the characteristics of low energy consumption and high efficiency for future communications, UAV communication can be quickly established and adjusted at low cost, which has been applied to ensure the communication of large-scale activities and disaster areas [17-19]. Considering the above advantages, many scientists and engineers have focused on the research of ISUAVTN. The authors of [20] discussed the energy efficient (EE) transmission of ISUAVTN, where total power constraint-based $\mathrm{BF}$ and per-antenna power constraint-based BF schemes were designed to maximize EE. In [21], a penalty functionbased algorithm was designed to maximize the sum rate of ISUAVTN with rate-splitting multiple access (RSMA), which can satisfy large-scale transmission. The authors of [22] considered a cache-enabled ISUAVTN, which can decrease transmission delay and accelerate the updates of documents. Liu et al. [23] established an uplink ISUAVTN with multiple users, and a BF scheme was designed to improve the system performance. The authors of [24] discussed the uplink massive access of ISUAVTN, where a space division multiple access (SDMA) scheme was proposed to optimize the ergodic sum rate.

Moreover, CR has been fully discussed in terrestrial wireless communication and has become a hot topic in ISTN. It can improve spectrum utilization by allowing secondary network (SN) to share the spectrum of primary network (PN), which is of great significance to the establishment of highly efficient network [25-27]. An EE-SE tradeoff index was designed to measure the performance of cognitive integrated satellite-vehicular networks in [27]; based on this, a power allocation scheme was developed. In [28], the authors investigated the performance of the cognitive ISTN with decode-and-forward (DF) protocol, in which the exact and asymptotic expressions of outage probability (OP) were derived. The authors of [29] established an overlay cognitive ISTN with multiple SNs, in which partial and opportunistic SN selection schemes were proposed to minimize the OP of PN. In [30], the authors investigated the outage performance of an underlay cognitive ISTN, where multiple primary users and HIs were considered. In [31], a BF scheme was developed to improve the security performance of a cognitive ISTN, where there existed multiple unknown eavesdroppers. The authors of [32] investigated the performance of an overlay cognitive ISTN with considering HIs, where an adaptive relay scheme was proposed to reduce OP.

In addition, the NOMA scheme can also be adopted to overcome spectrum shortage, especially in the scenario of large-scale ends, which can economize access resources.
In ISTN, the utilization of NOMA is able to save valuable time/frequency/code resources by sacrificing a little complexity of the receivers [33-35]. In [35], the authors applied bandwidth compression (BC) in NOMA-based ISTN to enhance spectrum utilization. The authors of [36] proposed a BF scheme based on an iterative algorithm to maximize the sum rate of NOMA-based ISTN, which considered the transmission quality of both satellite and terrestrial nodes. In [37], the authors discussed ergodic capacity (EC) of the uplink NOMA-based ISTN, but the exact closed-form expression of EC for the system was not provided. The authors of [38] considered a NOMA-based ISTN with multiple relays and HIs, in which secrecy outage probability (SOP) was derived to reveal the secrecy performance of two eavesdropping cases, namely, colluding case and noncolluding case. In [39], the authors investigated the performance of an underlay cognitive ISTN with NOMA scheme, in which the OP and EC were derived. The authors of [40] considered a NOMA-based cognitive ISTN with multiple SNs; the system performance was enhanced by adopting the partial SN selection scheme.

1.2. Main Contributions. Inspired by the abovementioned works, this paper considers a cognitive integrated satelliteUAV-terrestrial network (CISUAVTN) with NOMA scheme and multiple primary users, while all users are equipped with multiple antennas. Specifically, the major contributions of this paper are summarized as below:

(1) Firstly, a novel structure of overlay CISUAVTN with NOMA scheme and multiple primary users is built, where the communication of ISTN is assisted by secondary UAV. Besides, the satellite and UAV use the same frequency to enhance the SE.

(2) Secondly, considering the practical effects in signal propagation, such as rain attenuation and free path loss, the statistical properties of channels are provided. On this foundation, the exact expressions of OP and EC for both PN and SN are obtained.

(3) Thirdly, the asymptotic expression of OP for the two networks in high signal-to-noise ratio (SNR) regime is derived to obtain deeper opinions, based on which the diversity orders (DOs) for the two networks are also provided.

(4) Finally, to further comprehend the performance of our considered system, numerical results are provided. In addition, some observation results are also highlighted and analyzed.

1.3. Paper Organization. The composition of the remainder of the paper is shown as follows. Section 2 provides the system model, channel model, and problem formulation, while Section 3 investigates the statistical properties of channels and performance of our considered system. Besides, numerical results are given in Section 4, which analyzes the effects of critical parameters on system 
performance. Finally, the whole paper is summed up in Section 5.

Remark 1. $J_{n}(\cdot)$ denotes the $n$-order first-kind Bessel function. $|\cdot|$ is absolute values of complex number scalars, and the Frobenius-norm of vectors are denoted as $\|\cdot\|_{F} . E(\cdot)$ stands for the expectation operator and $(\cdot)^{H}$ represents conjugate transpose. $(\cdot)_{n}$ denotes the Pochhammer symbol (equation notation of [41]). $\mathbf{I}_{N \times M}$ is identity matrix with $N$ rows and $M$ columns and $\operatorname{di} \operatorname{ag}(\cdot, \cdot, \ldots, \cdot)$ is diagonal matrix. $G_{p, q}^{m, n}\left(t \mid \begin{array}{l}\left(a_{p}\right) \\ \left(b_{q}\right)\end{array}\right)$ is Meijer-G function (equation 9.301 of [41]).

\section{System Model and Problem Formulation}

As shown in Figure 1, an overlay CISUAVTN framework with NOMA scheme is established, where a PN and a SN exist. In the $\mathrm{PN}$, there are a source satellite $(A)$ and $K$ primary users $\left(\left\{C_{i}\right\}_{n=1}^{K}\right)$, while the SN includes a UAV transmitter $(B)$ and a terrestrial secondary user $(D)$. Considering some severe shadowing fading, it is assumed that $A$ cannot communicate with $C_{i}$ directly. Hence, overlay mode is adopted in our considered system, in which $B$ forwards the signals of $\mathrm{PN}$ and transmits the expected signals of $D$ simultaneously with DF protocol. Besides, each node works in half-duplex mode. In addition, both satellite and terrestrial nodes apply multiple antennas and all terrestrial nodes are located in the same satellite beam, while omnidirectional antenna is equipped at $B$ due to power constraint (it is assumed that perfect CSI can be achieved through feedback and training, and it has been adopted in DVES2). Moreover, the $K$ primary users are divided into $K / 2$ twouser pairs, which have been adopted in many former works $[33,40]$ (too many users will lead to heavy interference or high receiver complexity, so this paper adopts two-user pair scheme to balance performance gain and communication cost). Thus, we assume $C_{1}$ and $C_{2}$ are assigned to the same pair.

In general, it is assumed that the satellite-aerial link is modeled by Shadowed-Rician (SR) fading and aerial-terrestrial links experience Rayleigh fading.

2.1. Channel Model. To get closer to the actual transmission scenario, the impacts of rain attenuation, free space loss, and satellite antenna gain are considered in our system; thus, the channel coefficient between $A$ and $B$ is given by

$$
h_{a b}=V_{a b} g_{a b}
$$

where $g_{a b}$ is the random channel coefficient of SR fading, $V_{a b}=\sqrt{\left(\omega_{a b} \ell_{a b} \varepsilon_{a b} / \zeta_{a b}\right)}$, and $\zeta_{a b}$ is the rain attenuation coefficient distributed as $\mathscr{C} \mathcal{N}\left(\vartheta_{\zeta}, \sigma_{\zeta}^{2}\right)$, which undergoes lognormal random distribution.

Besides, $\omega_{a b}$ represents the influence of free space loss with

$$
\omega_{a b}=\left(\frac{v_{a b}}{f_{a b}}\right)^{2} \frac{1}{d_{a b}^{2}+d_{c}^{2}}
$$

where $v_{a b}=c / 4 \pi$ with $c$ denoting the speed of light, $f_{a b}$ represents the carrier frequency, $d_{a b} \approx 35786 \mathrm{~km}$, and $d_{c}$

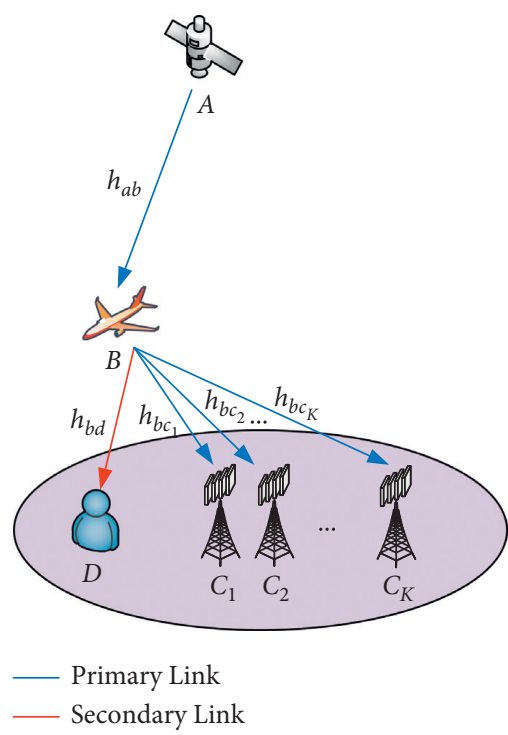

FIgURE 1: System model.

denotes the distance between the center of the satellite beam and the UAV.

Moreover,

$$
\ell_{a b} \simeq \begin{cases}\ell_{\max }-2.5 \times 10^{-3}\left(\frac{d_{b} \theta}{\eta}\right)^{2}, & 0^{\circ}<\theta<\theta_{1}, \\ 2+15 \log \frac{d_{b}}{\eta}, & \theta_{1}<\theta<\theta_{2}, \\ 32-25 \log \theta, & \theta_{2}<\theta<48^{\circ}, \\ -10, & 48^{\circ}<\theta<180^{\circ},\end{cases}
$$

denotes the antenna gain of UAV, where $\ell_{\max }$ is the maximum of $\ell_{a b}, d_{b}$ represents antenna diameter of $B, \eta$ is the signal wavelength, $\theta$ denotes the off-boresight angle, $\theta_{1}=\left(20 \eta / d_{b}\right) \sqrt{\ell_{\max }-\left(2+15 \log \left(d_{b} / \eta\right)\right)}, \quad$ and $\theta_{2}=15.85\left(\left(d_{b} / \eta\right)\right)^{-0.6}$.

In addition, the antenna gain of satellite is given by

$$
\varepsilon_{a b}=\varepsilon_{\max }\left[\frac{J_{1}(t)}{2 t}+36 \frac{J_{3}(t)}{t^{3}}\right]^{2}
$$

where $\varepsilon_{\max }$ is the maximum of $\varepsilon_{a b}$ and $t=2.70123\left(\sin \omega / \sin \omega_{3 \mathrm{~dB}}\right)$ with $\omega$ being the angle between UAV and satellite beam center and $\omega_{3 \mathrm{~dB}}$ denoting the $3 \mathrm{~dB}$ angle of $\omega$.

Furthermore, the $N_{e} \times 1$ channel vectors between UAV and terrestrial nodes are given by

$$
\mathbf{h}_{b e}=\mathscr{T}_{b e} \mathbf{g}_{b e},
$$

where the subscript $e=\left\{c_{1}, c_{2}, d\right\}$ represents destinations $C_{1}$, $C_{2}$, and $D, N_{e}$ is the number of antennas for user $e$, and $\mathbf{g}_{b e}$ denotes the channel complex vector of Rayleigh fading. $\mathscr{T}_{b e}$ can be represented as 


$$
\mathscr{T}_{b e}=\frac{1}{2}\left(20 \lg \lambda-10 \mathscr{I} \lg d_{b e}-20 \lg 4 \pi\right),
$$

where $\lambda$ is the center wavelength of the signal carrier, $\mathscr{I}$ is the path loss factor which ranges from 2 to 4 , and $d_{b e}$ is the distance between UAV and terrestrial nodes.

2.2. Signal Model. Because the system realizes communication with the help of UAV, the whole transmission process includes two time periods. In the first time period, $A$ transmits the integrated signal to $B$ by utilizing superposition coding technique (SCT), which is expected for terrestrial primary users and given by

$$
x_{a}=\sqrt{\alpha P_{a}} x_{1}+\sqrt{(1-\alpha) P_{a}} x_{2},
$$

where $\alpha$ is the power allocation coefficient of primary signal, $P_{a}$ denotes the transmit power of satellite, and $x_{1}$ and $x_{2}$ represent the target signal of $C_{1}$ and $C_{2}$, respectively, whose expectations are equal to 1 . Consequently, the received signal at $B$ can be expressed as (to simplify the analysis, hardware impairments and decoding errors have not been considered in this paper, which will be considered in our future work)

$$
y_{b}=h_{a b} x_{a}+n_{b}
$$

where $n_{b}$ is the additive white Gaussian noise (AWGN) with $\mathscr{C} \mathcal{N}\left(0, \sigma_{b}^{2}\right)$.

In the second time period, $B$ transmits the desired signal of $D$ and the decoded signal from $A$ to all terrestrial users simultaneously; the combined signal is given by

$$
s_{b}=\sqrt{\beta P_{b}}\left(\sqrt{\alpha} x_{1}+\sqrt{1-\alpha} x_{2}\right)+\sqrt{(1-\beta) P_{b}} x_{b},
$$

where $\beta$ is the power split coefficient of $B, P_{b}$ is the transmit power of the UAV, and $x_{b}$ is the signal $D$ expected with $E\left(\left|x_{b}\right|^{2}\right)=1$. Thus, we can obtain the received signal at terrestrial nodes as

$$
y_{b e}=\mathbf{w}_{b e}^{H} \mathbf{h}_{b e} s_{b}+\mathbf{w}_{b e}^{H} \mathbf{n}_{e},
$$

where $\mathbf{w}_{b e}=\mathbf{h}_{b e} /\left\|\mathbf{h}_{b e}\right\|_{F}$ is the $N_{e} \times 1$ receive $\mathrm{BF}$ weight vector and $\mathbf{n}_{e} \sim \mathscr{C} \mathcal{N}\left(0, \sigma_{e} \mathbf{I}_{N_{e} \times 1}\right)$ stands for the $N_{e} \times 1$ AWGN vector. In this study, we assume that $\left\|\mathbf{h}_{b c_{1}}\right\|_{F}<\left\|\mathbf{h}_{b c_{2}}\right\|_{F} ;$ thus, $\alpha \in(0.5,1)$.

2.3. Problem Formulation. According to the above illustration, the end-to-end signal-to-interference plus noise ratio (SINR) can be obtained.

Firstly, $B$ decodes $x_{1}$ with treating $x_{2}$ as interference; then, we can get the SINR of $x_{1}$ at $B$ as

$$
\gamma_{a b_{1}}^{R}=\frac{\alpha \gamma_{a b}}{(1-\alpha) \gamma_{a b}+1}
$$

where $\gamma_{a b}=\bar{\gamma}_{a b}\left|h_{a b}\right|^{2}$ and $\bar{\gamma}_{a b}=P_{a} / \sigma_{b}^{2}$ is the average SNR of $A-B$ link.

After that, $x_{1}$ is eliminated by $B$ and $x_{2}$ is decoded; thus, the SINR of $x_{2}$ at $B$ is given by

$$
\gamma_{a b_{2}}^{R}=(1-\alpha) \gamma_{a b}
$$

In the users' end, $C_{1}$ decodes its expected signal as regarding other signal as in-band interference; then, we can get the SINR by detecting $x_{1}$ at $C_{1}$ as

$$
\gamma_{b c_{1}}^{R}=\frac{\alpha \beta \gamma_{b c_{1}}}{\mu_{1} \gamma_{b c_{1}}+1},
$$

where $\quad \mu_{1}=(1-\alpha) \beta+1-\beta, \quad \gamma_{b c_{1}}=\bar{\gamma}_{b c_{1}}\left\|h_{b c_{1}}\right\|_{F}^{2}, \quad$ and $\bar{\gamma}_{b c_{1}}=P_{b} / \sigma_{c_{1}}^{2}$.

Recalling the NOMA scheme adopted in our proposed system, successive interference cancellation (SIC) is utilized in $C_{2}$; thus, $C_{2}$ decodes $x_{1}$ at first, and $x_{2}$ is treated as CCI. Therefore, the SINR of $x_{1}$ at $C_{2}$ can be represented as

$$
\gamma_{b c_{1 \rightarrow 2}}^{R}=\frac{\alpha \beta \gamma_{b c_{2}}}{\mu_{1} \gamma_{b c_{2}}+1},
$$

where $\gamma_{b c_{2}}=\bar{\gamma}_{b c_{2}}\left\|h_{b c_{2}}\right\|_{F}^{2}$ and $\bar{\gamma}_{b c_{2}}=P_{b} / \sigma_{c_{2}}^{2}$

Then, $x_{1}$ is cleared up by $C_{2}$ from $y_{b c_{2}}$ and $x_{2}$ is decoded; we can derive the SINR by discovering $x_{2}$ at $C_{2}$ as

$$
\gamma_{b c_{2}}^{R}=\frac{(1-\alpha) \beta \gamma_{b c_{2}}}{\mu_{2} \gamma_{b c_{2}}+1}
$$

where $\mu_{2}=1-\beta$.

In addition, the SINR of $x_{b}$ at $D$ is given by

$$
\gamma_{b d}^{R}=\frac{(1-\beta) \gamma_{b d}}{\beta \gamma_{b d}+1},
$$

where $\gamma_{b d}=\bar{\gamma}_{b d}\left\|h_{b d}\right\|_{F}^{2}$ and $\bar{\gamma}_{b d}=P_{b} / \sigma_{d}^{2}$.

\section{Performance Analysis}

In this section, the statistical properties of SR fading and Rayleigh fading are provided. On this basis, the exact expressions of OP for both primary network and secondary network are derived. Besides, we derive the asymptotic OP of the two networks in high SNR regime to obtain further insights. Finally, the exact ECs of the two networks are obtained.

3.1. Statistical Properties of Channels. Firstly, the probability distribution function (PDF) and cumulative distribution function (CDF) of SR fading and Rayleigh fading are obtained. From [42], the PDF of $g_{a b}$ is given by

$$
f_{\left|g_{a b}\right|^{2}}(x)=\alpha_{a b} e_{1}^{-\beta_{a b} x} F_{1}\left(m_{a b} ; 1 ; \delta_{a b} x\right),
$$

where $\alpha_{a b} \triangleq\left(1 / 2 b_{a b}\right)\left(2 b_{a b} m_{a b} /\left(2 b_{a b} m_{a b}+\Omega_{a b}\right)\right)^{m_{a b}}, \beta_{a b} \triangleq$ $\left(1 / 2 b_{a b}\right)$, and $\delta_{a b} \triangleq\left(\Omega_{a b} / 2 b_{a b}\left(2 b_{a b} m_{a b}+\Omega_{a b}\right)\right)$. $m_{a b}$ represents the Nakagami-m parameter which is always greater than 0 , and $2 b_{a b}$ is the average power of the multi-path part, while $\Omega_{a b}$ is that of line of sight (LOS) part.

Under the situation that $m_{a b}$ is an integer, by using equation 07.20.03.009.01 of [43] and equation 07.02.03.0014.01 of [43], ${ }_{1} F_{1}\left(m_{a b} ; 1 ; \delta_{a b} x\right)$ is represented as 
${ }_{1} F_{1}\left(m_{a b} ; 1 ; \delta_{a b} x\right)=e^{-\delta_{a b} x} \sum_{n=0}^{m_{a b}-1} \frac{\left(-\delta_{a b}\right)^{n}\left(1-m_{a b}\right)_{n}}{(n !)^{2}} x^{n}$.

According to (17) and (18), the PDF of $g_{a b}$ can be rewritten as

$f_{\left|g_{a b}\right|^{2}}(x)=\alpha_{a b} e^{-\left(\beta_{a b}-\delta_{a b}\right) x} \sum_{n=0}^{m_{a b}-1} \frac{\left(-\delta_{a b}\right)^{n}\left(1-m_{a b}\right)_{n}}{(n !)^{2}} x^{n}$.

From (1) and $\gamma_{a b}=\bar{\gamma}_{a b}\left|h_{a b}\right|^{2}$, we can get the PDF of $\gamma_{a b}$ as

$$
f_{\gamma_{a b}}(x)=\alpha_{a b} \sum_{n=0}^{m_{a b}-1} \xi(n) x^{n} e^{-\Delta_{a b} x},
$$

where $\Delta_{a b}=\left(\beta_{a b}-\delta_{a b}\right) / \bar{\gamma}_{a b}$ and

$$
\xi(n)=\frac{\left(-\delta_{a b}\right)^{n}\left(1-m_{a b}\right)_{n}}{(n !)^{2}\left(V_{a b}^{2} \bar{\gamma}_{a b}\right)^{n+1}} .
$$

By utilizing equation 3.351.2 of [41], the CDF of $\gamma_{a b}$ is given by

$$
F_{\gamma_{a b}}(x)=1-\alpha_{a b} \sum_{n=0}^{m_{a b}-1} \sum_{t=0}^{n} \frac{n ! \xi(n)}{t ! \Delta_{a b}^{n-t+1}} x^{t} e^{-\Delta_{a b} x} .
$$

From $[39,44]$, the PDF and CDF of $\gamma_{b e}$ can be expressed as, respectively, $f_{\gamma_{b e}}(x)=\sum_{i=1}^{\rho\left(\Lambda_{b e}\right)} \sum_{j=1}^{\delta_{i}\left(\Lambda_{b e}\right)} \Xi_{i, j}\left(\Lambda_{b e}\right) \frac{\lambda_{\langle i\rangle}^{-j}}{(j-1) !} x^{j-1} e^{-\left(x / \lambda_{\langle i\rangle}\right)}$

$$
F_{\gamma_{b e}}(x)=1-\sum_{i=1}^{\rho\left(\Lambda_{b e}\right)} \sum_{j=1}^{\delta_{i}} \sum_{t=0}^{\left(\Lambda_{b e}\right)} \frac{j-1}{\Xi_{i, j}\left(\Lambda_{b e}\right)}\left(\frac{x}{\lambda_{\langle i\rangle}}\right)^{t} e^{-\left(x / \lambda_{\langle i}\right)},
$$

where $\left\{\lambda_{i}\right\}_{i=1}^{N_{e}}$ is the average SNR of aerial-terrestrial links, $\Lambda_{b e}=\operatorname{diag}\left(\lambda_{1}, \lambda_{2}, \ldots, \lambda_{N_{e}}\right), \rho\left(\Lambda_{b e}\right)$ denotes the amount of different diagonal elements of $\Lambda_{b e}$, $\lambda_{\left\langle\rho\left(\Lambda_{b e}\right)\right\rangle}<\cdots<\lambda_{\langle 2\rangle}<\lambda_{\langle 1\rangle}$ are the different diagonal elements in ascending sequence, $\delta_{i}\left(\Lambda_{b e}\right)$ is the multiplicity of $\lambda_{\langle i\rangle}$, and $\Xi_{i, j}\left(\Lambda_{b e}\right)$ represents the $(i, j)$ th characteristic coefficient of $\Lambda_{b e}$.

3.2. OP. OP can well evaluate the performance of the system, and in this paper, we define the OP as the probability of the instantaneous capacity for any nodes lower than its excepted capacity.

3.2.1. OP of PN. From the definition of OP, the OP of PN can be expressed as

$$
\begin{aligned}
P_{\text {out }}^{p}= & 1-\operatorname{Pr}\left[\frac{1}{2} \log _{2}\left(1+\gamma_{a b_{1}}^{R}\right) \geq R_{1}, \frac{1}{2} \log _{2}\left(1+\gamma_{a b_{2}}^{R}\right) \geq R_{2}\right] \operatorname{Pr}\left[\frac{1}{2} \log _{2}\left(1+\gamma_{b c_{1}}^{R}\right) \geq R_{1}, \frac{1}{2} \log _{2}\left(1+\gamma_{b c_{1} \rightarrow 2}^{R}\right) \geq R_{1}\right] \\
& \times \operatorname{Pr}\left[\frac{1}{2} \log _{2}\left(1+\gamma_{b c_{2}}^{R}\right) \geq R_{2}\right] .
\end{aligned}
$$

Then, by letting $\gamma_{1}=2^{2 R_{1}}-1$ and $\gamma_{2}=2^{2 R_{2}}-1$, (25) can be rewritten as

$P_{\text {out }}^{p}=1-\left[1-F_{\gamma_{a b}}\left(\tau_{\max }\right)\right]\left[1-F_{\gamma_{b c_{1}}}\left(\varphi_{1}\right)\right]\left[1-F_{\gamma_{b c_{2}}}\left(\varphi_{\max }\right)\right]$,

$P_{\text {out }}^{P}=1-\operatorname{Pr}\left(\gamma_{a b_{1}}^{R} \geq \gamma_{1}, \gamma_{a b_{2}}^{R} \geq \gamma_{2}\right) \operatorname{Pr}\left(\gamma_{b c_{1}}^{R} \geq \gamma_{1}, \gamma_{b c_{1 \rightarrow 2}}^{R} \geq \gamma_{1}, \gamma_{b c_{2}}^{R} \geq \gamma_{2}\right)$.

By substituting (11)-(15) into (26), we can obtain where $\quad \tau_{\max }=\max \left(\tau_{1}, \tau_{2}\right), \quad \tau_{1}=\gamma_{1} /\left(\alpha-\gamma_{1}(1-\alpha)\right)$, $\tau_{2}=\gamma_{2} /(1-\alpha), \quad \varphi_{\max }=\max \left(\varphi_{1}, \varphi_{2}\right), \varphi_{1}=\gamma_{1} /\left(\alpha \beta-\mu_{1} \gamma_{1}\right)$, and $\varphi_{2}=\gamma_{2} /\left((1-\alpha) \beta-\mu_{2} \gamma_{2}\right)$.

At last, by taking (22) and (24) into (27), the final expression of OP for $\mathrm{PN}$ is obtained as

$$
\begin{aligned}
P_{\text {out }}^{p}= & -\alpha_{a b} \sum_{n=0}^{m_{a b}-1} \sum_{t=0}^{n} \frac{n ! \xi(n)}{t ! \Delta_{a b}^{n-t+1}} \tau_{\max }^{t} e^{-\Delta_{a b} \tau_{\max }} \sum_{i_{1}=1}^{\rho\left(\Lambda_{b c_{1}}\right)} \sum_{j_{1}=1}^{\delta_{i_{1}}\left(\Lambda_{b c_{1}}\right)} \sum_{t_{1}=0}^{j_{1}-1} \frac{\Xi_{i_{1}, j_{1}}\left(\Lambda_{b c_{1}}\right)}{t_{1} !}\left(\frac{\varphi_{1}}{\left.\lambda_{\left\langle i_{1}\right\rangle}\right\rangle}\right)^{t_{1}} e^{-\left(\varphi_{1} / \lambda_{\left\langle i_{1}\right\rangle}\right)} \\
& \times \sum_{i_{2}=1}^{\rho\left(\Lambda_{b c_{2}}\right)} \sum_{j_{2}=1}^{\delta_{i_{2}}\left(\Lambda_{b c_{1}}\right)} \sum_{t_{2}=0}^{j_{j_{2}-1}} \frac{\Xi_{i_{2}, j_{2}}\left(\Lambda_{b c_{1}}\right)}{t_{2} !}\left(\frac{\varphi_{\max }}{\lambda_{\left\langle i_{2}\right\rangle}}\right)^{t_{2}} e^{-\left(\varphi_{\max } / \lambda_{\left\langle i_{2}\right\rangle}\right)} .
\end{aligned}
$$


3.2.2. OP of SN. Similar to the derivation of OP for PN, the $\mathrm{OP}$ for $\mathrm{SN}$ is given by

$$
\begin{aligned}
P_{\text {out }}^{S} & =1-\operatorname{Pr}\left[\frac{1}{2} \log _{2}\left(1+\gamma_{a b_{1}}^{R}\right) \geq R_{1}, \frac{1}{2} \log _{2}\left(1+\gamma_{a b_{2}}^{R}\right) \geq R_{2}\right] \operatorname{Pr}\left[\frac{1}{2} \log _{2}\left(1+\gamma_{b d}^{R}\right) \geq R_{d}\right] \\
& =1-\left[1-F_{\gamma_{a b}}\left(\tau_{\max }\right)\right]\left[1-F_{\gamma_{b d}}\left(\varphi_{d}\right)\right],
\end{aligned}
$$

where $\varphi_{d}=\gamma_{d} /\left(1-\beta-\beta \gamma_{d}\right)$ and $\gamma_{d}=2^{2 R_{d}}-1$. By utilizing

(22) and (24) into (29), we can obtain

$$
P_{\text {out }}^{s}=1-\alpha_{a b} \sum_{n=0}^{m_{a b}-1} \sum_{t=0}^{n} \frac{n ! \xi(n)}{t ! \Delta_{a b}^{n-t+1}} \tau_{\max }^{t} e^{-\Delta_{a b} \tau_{\max }} \times \sum_{i=1}^{\rho\left(\Lambda_{b d}\right)} \sum_{j=1}^{\delta_{i}\left(\Lambda_{b d}\right)} \sum_{t_{1}=0}^{j-1} \frac{\Xi_{i, j}\left(\Lambda_{b d}\right)}{t_{1} !}\left(\frac{\varphi_{d}}{\lambda_{\langle i\rangle}}\right)^{t_{1}} e^{-\left(\varphi_{d} / \lambda_{\langle i\rangle}\right)}
$$

3.3. Asymptotic OP. To achieve deeper insights, we discuss the asymptotic OP in high SNR regime.

3.3.1. Asymptotic OP of PN. Firstly, in high SNR regime, the CDF of $\gamma_{a b}$ and $\gamma_{b e}$ are approximately represented as

$$
\begin{gathered}
F_{\gamma_{a b}}(x) \triangleq \frac{\alpha_{a b} x}{\bar{\gamma}_{a}}, \\
F_{\gamma_{b e}}(x) \triangleq \frac{1}{N_{e} !}\left(\frac{x}{\bar{\gamma}_{b}}\right)^{N_{e}} .
\end{gathered}
$$

By substituting (31) and (32) into (27), the asymptotic expression of OP for $\mathrm{PN}$ is derived as

$$
P_{\text {out }}^{p \rightarrow \infty}= \begin{cases}\frac{\alpha_{a b} \tau_{\max }}{\bar{\gamma}_{a}}, & N_{c_{1}}>1, N_{c_{2}}>1, \\ \frac{\alpha_{a b} \tau_{\max }}{\bar{\gamma}_{a}}+\frac{1}{N_{c_{1}} !}\left(\frac{\varphi_{1}}{\bar{\gamma}_{b}}\right)^{N_{c_{1}}}, & N_{c_{1}}=1, N_{c_{2}}>1, \\ \frac{\alpha_{a b} \tau_{\max }}{\bar{\gamma}_{a}}+\frac{1}{N_{c_{2}} !}\left(\frac{\varphi_{\max }}{\bar{\gamma}_{b}}\right)^{N_{c_{2}},} & N_{c_{1}}>1, N_{c_{2}}=1, \\ \frac{\alpha_{a b} \tau_{\max }}{\bar{\gamma}_{a}}+\frac{1}{N_{c_{1}} !}\left(\frac{\varphi_{1}}{\bar{\gamma}_{b}}\right)^{N_{c_{1}}}+\frac{1}{N_{c_{2}} !}\left(\frac{\varphi_{\max }}{\bar{\gamma}_{b}}\right)^{N_{c_{2}}}, & N_{c_{1}}=1, N_{c_{2}}>1 .\end{cases}
$$

From (33), the DO of PN can be clearly obtained as $\mathrm{DO}_{p}=\min \left(1, N_{c_{1}}, N_{c_{2}}\right)$.

3.3.2. Asymptotic OP of $S N$. With the similar method to derive asymptotic $\mathrm{OP}$ of $\mathrm{PN}$, we can get the asymptotic expression of OP for $\mathrm{SN}$ as

$$
P_{\text {out }}^{s \rightarrow \infty}= \begin{cases}\frac{\alpha_{a b} \tau_{\max }}{\bar{\gamma}_{a}}, & N_{d}>1, \\ \frac{\alpha_{a b} \tau_{\max }}{\bar{\gamma}_{a}}+\frac{1}{N_{c_{1}} !}\left(\frac{\varphi_{1}}{\bar{\gamma}_{b}}\right)^{N_{c_{1}}}, & N_{d}=1 .\end{cases}
$$

Besides, the $\mathrm{DO}$ of $\mathrm{SN}$ is $\mathrm{DO}_{s}=\min \left(1, N_{d}\right)$.

3.4. EC. EC is also a momentous index in transmission system, which is defined as the time average of the maximal transmission capacity from the transmitter to the receiver under all fading conditions.

3.4.1. EC of PN. The EC of PN is defined as the minimal EC of the satellite-aerial link and aerial-terrestrial links (equation 19 of [45]), which can be expressed as

$$
\mathrm{EC}=\min \left(\mathrm{EC}_{a b}, \mathrm{EC}_{b c}\right),
$$


where $\mathrm{EC}_{a b}$ and $\mathrm{EC}_{b c}$ are the $\mathrm{EC}$ of satellite-aerial link and aerial-terrestrial links, respectively. To get the final expression of $\mathrm{EC}$ for $\mathrm{PN}$, we should derive $\mathrm{EC}_{a b}$ and $\mathrm{EC}_{b c}$ at first.

Theorem 1. The exact expression of $E C_{a b}$ can be expressed as

$$
\mathrm{EC}_{a b}=\frac{1}{2 \ln 2} \alpha_{a b} \sum_{n=0}^{m_{a b}-1} \frac{\xi(n)}{\Delta_{a b}^{n+1}} G_{2,3}^{3,1}\left(\begin{array}{cc}
\Delta_{a b} \mid & 0,1 \\
1+n, 1,1
\end{array}\right)
$$

Proof. See Appendix A.

Theorem 2. The exact expression of $E C_{b c}$ can be expressed as

$$
\begin{aligned}
\mathrm{EC}_{b c}=\frac{1}{2 \ln 2}\left\{\sum_{i_{1}=1}^{\rho\left(\Lambda_{b c_{1}}\right)} \sum_{j_{1}=1}^{\delta_{i_{1}}\left(\Lambda_{b c_{1}}\right)} \Xi_{i_{1}, j_{1}}\left(\Lambda_{b c_{1}}\right) \frac{1}{\left(j_{1}-1\right) !}\left[G_{2,3}^{3,1}\left(\left.\frac{1}{\lambda_{\left\langle i_{1}\right\rangle}}\right|_{j_{1}, 0,0} ^{0,1}\right)-G_{2,3}^{3,1}\left(\left.\frac{1}{\mu_{1} \lambda_{\left\langle i_{1}\right\rangle}}\right|_{j_{1}, 0,0} ^{0,1}\right)\right]\right. \\
\left.\quad+\sum_{i_{2}=1}^{\rho\left(\Lambda_{b c_{2}}\right)} \sum_{j_{2}=1}^{\delta_{i_{2}}\left(\Lambda_{b c_{2}}\right)} \Xi_{i_{2}, j_{2}}\left(\Lambda_{b c_{2}}\right) \frac{1}{\left(j_{2}-1\right) !}\left[G_{2,3}^{3,1}\left(\frac{1}{\lambda_{\left\langle i_{2}\right\rangle}} \mid \begin{array}{c}
0,1 \\
j_{2}, 0,0
\end{array}\right)-G_{2,3}^{3,1}\left(\left.\frac{1}{\mu_{2} \lambda_{\left\langle i_{2}\right\rangle}}\right|_{j_{2}, 0,0} ^{0,1}\right)\right]\right\} .
\end{aligned}
$$

Proof. See Appendix B.

Finally, by taking (36) and (37) into (35), we can obtain the final EC of PN.

\subsubsection{EC of $S N$}

Theorem 3. The exact expression of EC for $S N$ can be represented as

$$
\mathrm{EC}_{S N}=\frac{1}{2 \ln 2}\left\{\sum_{i_{d}=1}^{\rho\left(\Lambda_{b d}\right)} \sum_{j_{d}=1}^{\delta_{i_{d}}\left(\Lambda_{b d}\right)} \Xi_{i_{d}, j_{d}}\left(\Lambda_{b d}\right) \frac{1}{\left(j_{d}-1\right) !}\left[G_{2,3}^{3,1}\left(\begin{array}{cc}
1 & 0,1 \\
\lambda_{\left\langle i_{d}\right\rangle} & j_{d}, 0,0
\end{array}\right)-G_{2,3}^{3,1}\left(\begin{array}{cc}
1 & 0,1 \\
\beta \lambda_{\left\langle i_{d}\right\rangle} & \\
j_{d}, 0,0
\end{array}\right)\right]\right\}
$$

Proof. The proof is similar to Appendix B, and the detailed process is omitted here.

\section{Numerical Results}

To testify the validity of our derivations, the simulation results are offered in this section. Particularly, we assume that $\bar{\gamma}_{a b}=\bar{\gamma}_{b c_{1}}=\bar{\gamma}_{b c_{2}}=\bar{\gamma}_{b d}=\bar{\gamma}, \sigma_{a b}^{2}=\sigma_{b c_{1}}^{2}=\sigma_{b c_{2}}^{2}=\sigma_{b d}^{2}=\sigma^{2}$, and $N_{c_{1}}=N_{c_{2}}=N_{d}=N$. Besides, the system and SR fading parameters $\left(m_{a b}, b_{a b}, \Omega_{a b}\right)$ are provided in Table 1, in detail [29].

Firstly, from the simulation results, we can clearly find that the Monte Carlo (MC) simulations results are in accordance with our theoretical results; thus, the effectiveness of the analysis in Section 3 is demonstrated. Moreover, the exact and asymptotic OP coincides briefly in high SNR regime, which indicates that our asymptotic analysis is correct.

Figure 2 shows that $\mathrm{OP}$ of $\mathrm{PN}$ versus different numbers of antenna $N$ with $R_{1}=R_{2}=0.5 \mathrm{~b} /(\mathrm{s} / \mathrm{Hz})$, $\alpha=0.67$, and $\beta=0.9$. It can be easily seen that worse channel condition lead to the deterioration of outage performance. Besides, OP decreases with the number of antenna; this is because that more antennas will enhance the receiving capacity of the users. In addition, the increasing of average SNR will improve the outage performance.
Figure 3 depicts that $\mathrm{OP}$ of $\mathrm{PN}$ versus different power allocation coefficients $\alpha$ with setting $\bar{\gamma}=20 \mathrm{~dB}$, $R_{1}=R_{2}=0.5 \mathrm{~b} /(\mathrm{s} / \mathrm{Hz}), N=3$, and $\beta=0.9$. It is interesting that outage performance first enhances and then decreases with the growth of $\alpha$. This is due to the fact that the OP can achieve minimum by adjusting power allocation coefficient.

Figure 4 plots that OP of PN versus different target rates $R_{1}$ and $R_{2}$ with $\alpha=0.67, N=3$, and $\beta=0.9$. It can be clearly observed that OP degrades with target rates. This is because that the decrease in system requirements will reduce the OP.

Figure 5 illustrates OP of SN versus different number of antenna $N$ with setting $R_{d}=0.5(\mathrm{~b} /(\mathrm{s} / \mathrm{Hz}))$ and $\beta=0.9$. We can find that the outage performance enhance in pace with $N$, which is similar to the results of $\mathrm{PN}$.

Figure 6 shows that EC of PN versus different numbers of antenna $N$ with $\alpha=0.67$ and $\beta=0.9$. Obviously, better channel condition can improve the EC of system, which is coincident with outage performance. Furthermore, EC increases with $\bar{\gamma}$, but the upward trend slows down and curves of different channel conditions coincide in high SNR regime. The reason is that the EC of second hop lower than that of first hop when average SNR is high, and $\mathrm{EC}_{b c}$ is independent of SR fading parameters. In addition, the growth of $N$ will enhance the system performance in high SNR regime, while the curves of 
TABLE 1: Simulation parameters.

Parameter name

Satellite

Rain attenuation $\left(\vartheta_{\zeta}, \sigma_{\zeta}^{2}\right)$

Carrier frequency $f_{a b}$

$3 \mathrm{~dB}$ angle $\theta_{3 \mathrm{~dB}}$

Maximal satellite gain $\varepsilon_{\max }$

Maximal UAV gain $\ell_{\max }$

Carrier bandwidth $B$

Noise temperature $T$

Variance of AWGN $\sigma^{2}$

Frequent heavy shadowing (FHS)

Average shadowing (AS)

Infrequent light shadowing (ILS)
Parameter value

GEO

$(-3.125,1.591)$

$2 \mathrm{GHz}$

$0.4^{\circ}$

$48 \mathrm{~dB}$

$4 \mathrm{~dB}$

$15 \mathrm{MHz}$

$300^{\circ}$

1

$(1,0.063,0.0007)$

$(5,0251,0.279)$

$(10,0.158,1.29)$
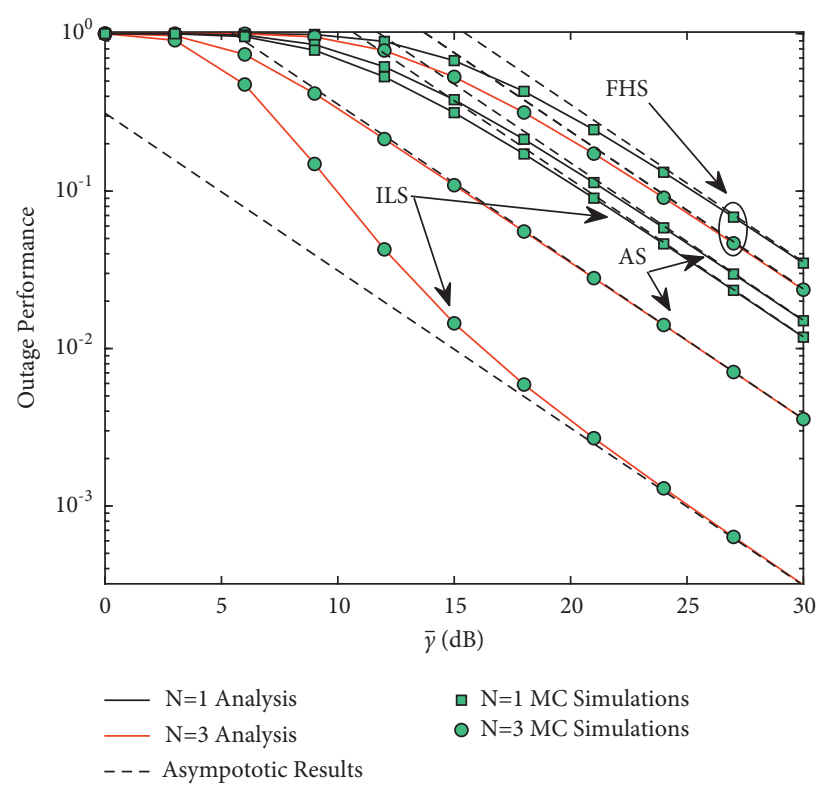

FIGURE 2: OP of PN versus different numbers of antenna, $N$.

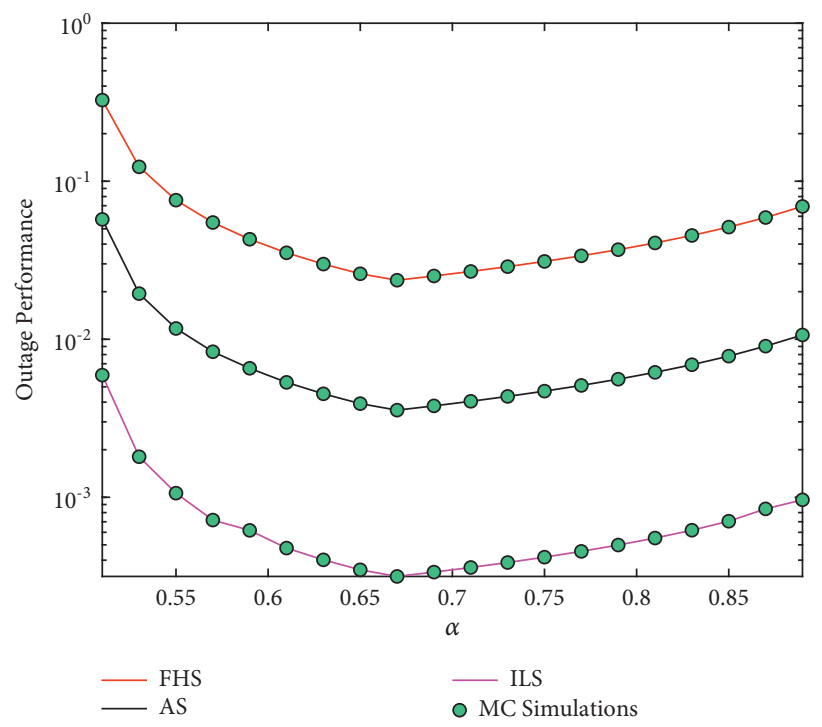

FIgURE 3: OP of PN versus different power allocation coefficients, $\alpha$. 


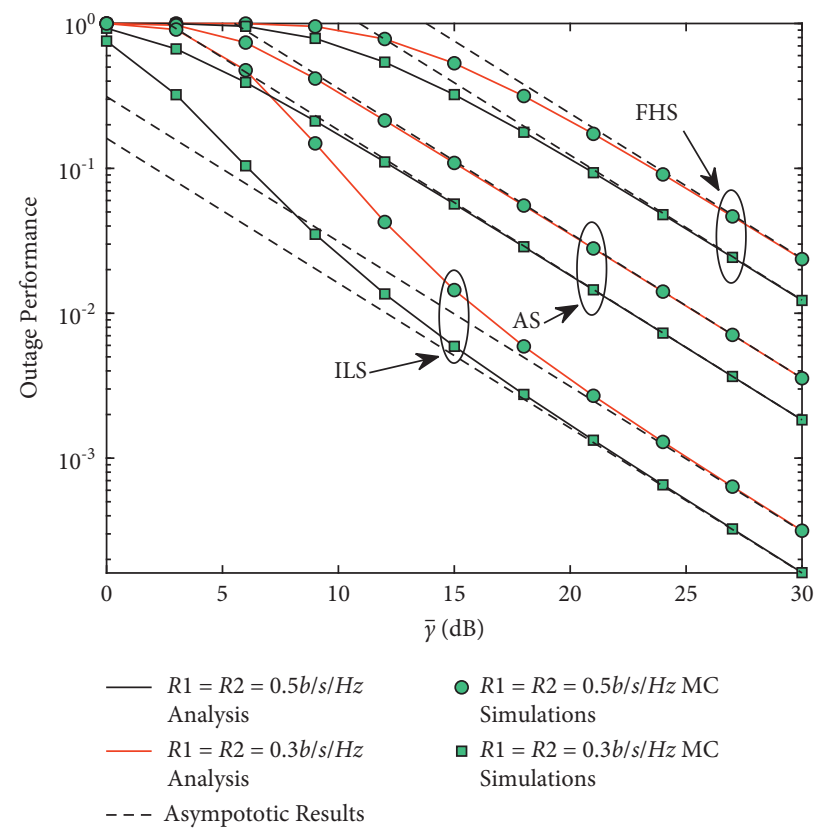

Figure 4: OP of PN versus different target rates, $R_{1}$ and $R_{2}$.

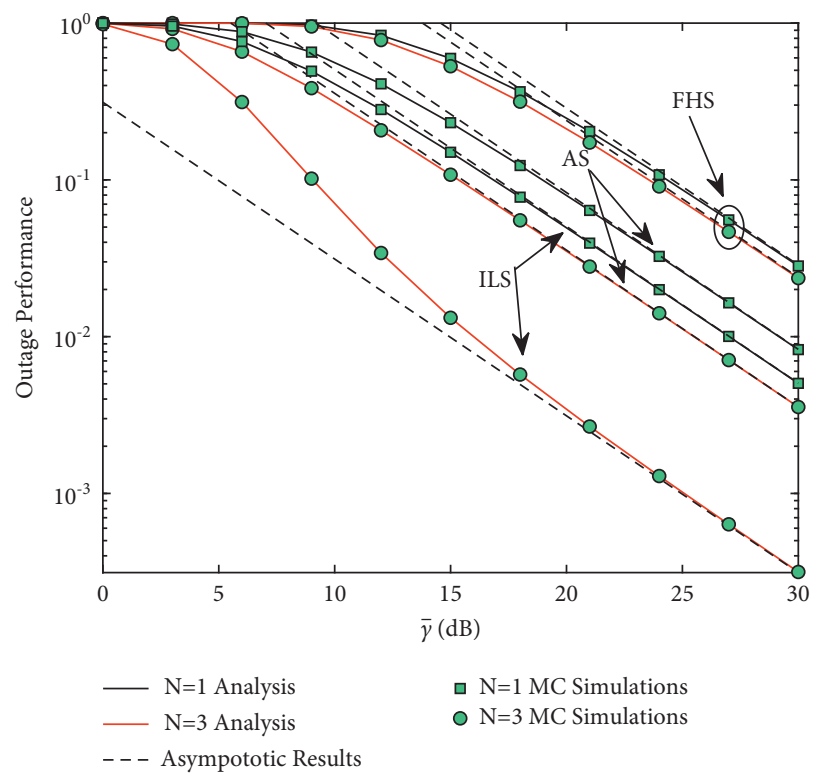

FIGURE 5: OP of SN versus different numbers of antenna, $N$.

different numbers of antenna overlap under low SNRs. It is because the changes of $N$ have no impact on $\mathrm{EC}_{a b}$, which is higher than $\mathrm{EC}_{b c}$ in low SNR regime.

Figure 7 depicts that EC of PN versus different power allocation coefficients $\alpha$ with setting $\bar{\gamma}=20 \mathrm{~dB}$ and $\beta=0.9$. It can be clearly found that EC increases in pace with $\alpha$ under AS and ILS and is a fixed value under FHS. This is because the final ECs under AS and ILS are taken as $\mathrm{EC}_{b c}$ in $\bar{\gamma}=20 \mathrm{~dB}$, while that under FHS is taken as $\mathrm{EC}_{a b}$.
Figure 8 plots that EC of PN versus different power spilt coefficients $\beta$ with $\bar{\gamma}=20 \mathrm{~dB}$ and $\alpha=0.67$. We can observe that system performance enhances with the growth of $\beta$, which represents more power of $B$ is allocated to transmit primary signal.

Figure 9 illustrates EC of SN versus different numbers of antenna $N$. As expected, EC increases with the number of antenna. Contrary to $\mathrm{PN}, \mathrm{EC}$ of $\mathrm{SN}$ degrades with the improvement of $\beta$. 


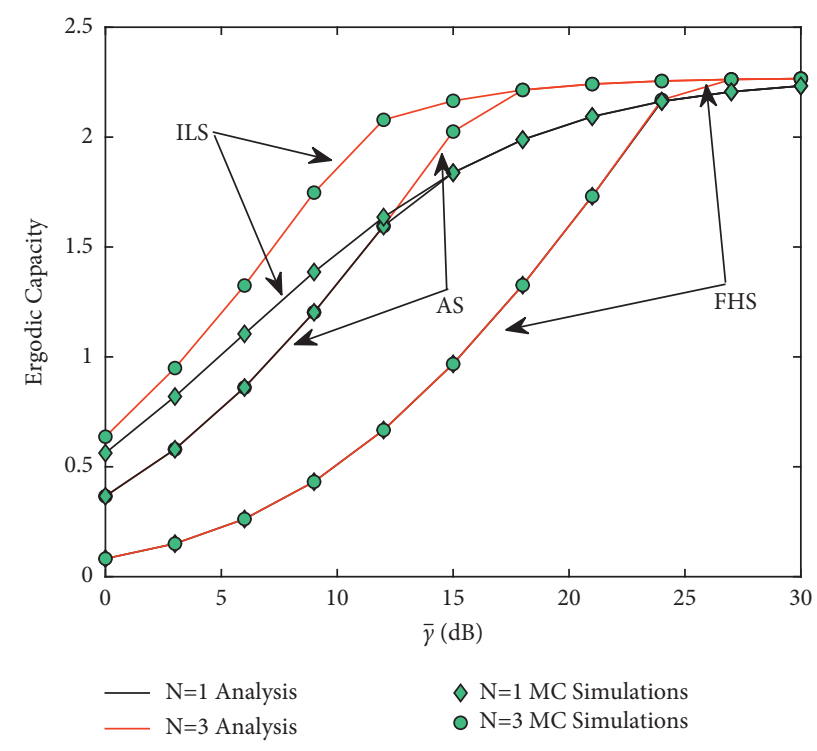

Figure 6: EC of PN versus different numbers of antenna $N$.

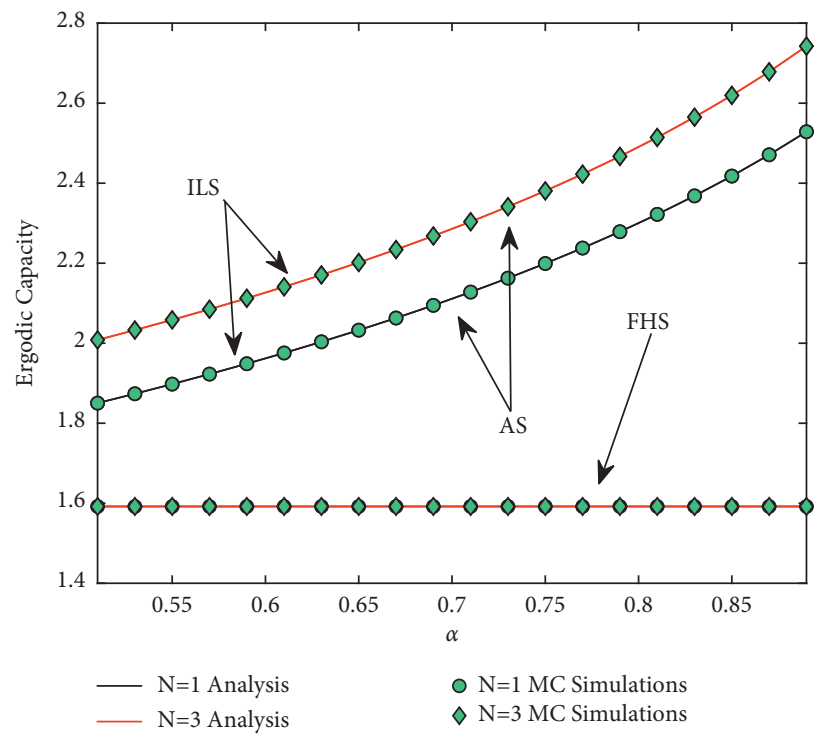

Figure 7: EC of PN versus different power allocation coefficients $\alpha$. 


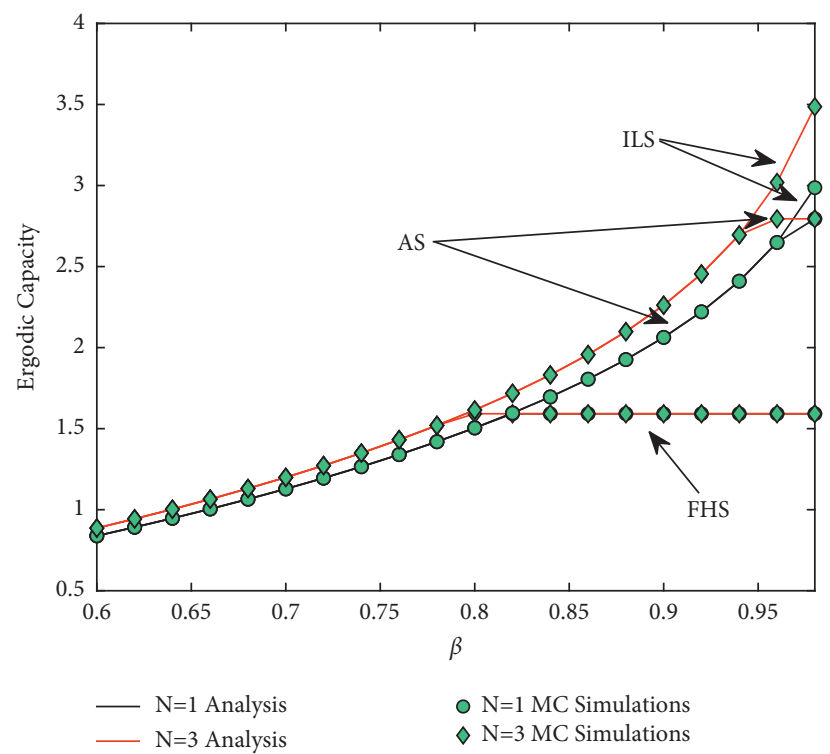

Figure 8: EC of PN versus different power spilt coefficients $\beta$.

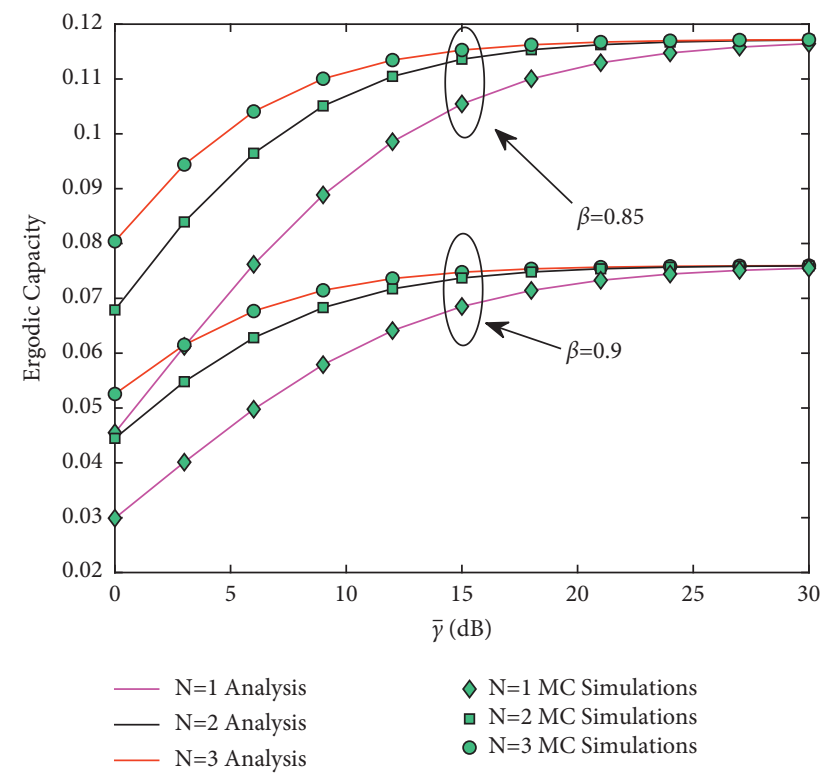

FIGURE 9: EC of SN versus different numbers of antenna, $N$.

\section{Conclusions}

In this paper, a CISUAVTN with NOMA scheme and multiple primary users was established, in which a secondary UAV forwarded the signal of satellite to terrestrial users in the overlay mode. Considering the actual transmission loss, the system performance was discussed. Particularly, the closed-form expressions of OP for both PN and SN were provided. Besides, the asymptotic OP and DOs were derived to gain additional views. Moreover, the exact EC of the two networks were derived. According to numerical results, the better channel condition and larger number of antenna can enhance the system performance. In addition, the power allocation coefficient and power spilt coefficient can be optimized to get superior performance of $\mathrm{PN}$ and balance the performance of the two networks, respectively.

\section{Appendix}

\section{A. The Proof of (36)}

Recalling the definition of $\mathrm{EC}, \mathrm{EC}_{a b}$ can be expressed as

$$
\mathrm{EC}_{a b}=\frac{1}{2}\left\{E\left[\log _{2}\left(1+\gamma_{a b_{1}}^{R}\right)\right]+E\left[\log _{2}\left(1+\gamma_{a b_{2}}^{R}\right)\right]\right\} .
$$

By utilizing (11) and (12) into (A.1), we can obtain

$$
\begin{aligned}
\mathrm{EC}_{a b} & =\frac{1}{2 \ln 2} E\left[\ln \left(1+\gamma_{a b}\right)\right] \\
& =\frac{1}{2 \ln 2} \int_{0}^{\infty} \ln (1+x) f_{\gamma_{a b}}(x) \mathrm{d} x
\end{aligned}
$$

Then, we utilize Meijer-G function to solve the above integral formula. From equation 8.4.6.5 of [46], we can obtain

$$
\ln (1+x)=G_{2,2}^{1,2}\left(\begin{array}{cc}
x \mid & 1,1 \\
& 1,0
\end{array}\right)
$$

By taking (A.3) and (20) into (A.4), we can obtain

$$
\mathrm{EC}_{a b}=\frac{1}{2 \ln 2} \int_{0}^{\infty} G_{2,2}^{1,2}\left(\begin{array}{c}
1,1 \\
x \mid \\
1,0
\end{array}\right) \alpha_{a b} \sum_{n=0}^{m_{a b}-1} \xi(n) x^{n} e^{-\Delta_{a b} x} \mathrm{~d} x
$$

According to equation 2.24.3.1 of [46], we can obtain 


$$
\mathrm{EC}_{a b}=\frac{1}{2 \ln 2} \alpha_{a b} \sum_{n=0}^{m_{a b}-1} \frac{\xi(n)}{\Delta_{a b}^{n+1}} G_{3,2}^{1,3}\left(\left.\frac{1}{\Delta_{a b}}\right|^{-n, 1,1}\right)
$$

To avoid the case of $\Delta_{a b}=0$, from equation 8.2.2.14 of [46], we can obtain

$$
G_{p, q}^{m, n}\left(\begin{array}{c}
\left(a_{p}\right) \\
t \mid \\
\left(b_{q}\right)
\end{array}\right)=G_{q, p}^{n, m}\left(\begin{array}{c}
1 \\
\frac{1}{t}\left(1-b_{q}\right) \\
\left(1-a_{p}\right)
\end{array}\right) .
$$

From (A.5) and (A.6), (36) can be obtained. The proof of Theorem 1 is completed.

\section{B. The Proof of (37)}

Similar to $\mathrm{EC}_{a b}, \mathrm{EC}_{b c}$ is given by

$$
\begin{aligned}
\mathrm{EC}_{b c} & =\frac{1}{2}\left\{E\left[\log _{2}\left(1+\gamma_{b c_{1}}^{R}\right)\right]+E\left[\log _{2}\left(1+\gamma_{b c_{1 \rightarrow 2}}^{R}\right)\right]+E\left[\log _{2}\left(1+\gamma_{b c_{2}}^{R}\right)\right]\right\} \\
& =\frac{1}{2 \ln 2}\left\{E\left[\ln \left(1+\gamma_{b c_{1}}\right)\right]+E\left[\ln \left(1+\gamma_{b c_{2}}\right)\right]-E\left[\ln \left(1+\mu_{1} \gamma_{b c_{1}}\right)\right]-E\left[\ln \left(1+\mu_{2} \gamma_{b c_{2}}\right)\right]\right\}
\end{aligned}
$$

Firstly, when $z=B x$, we can obtain

$$
f_{z}(z)=\frac{1}{B} f_{x}\left(\frac{x}{B}\right)
$$

$$
f_{y}(y)=\sum_{i=1}^{\rho\left(\Lambda_{b c_{1}}\right)} \sum_{j=1} \delta_{i}\left(\Lambda_{b c_{1}}\right)\left(\frac{1}{\mu_{1}}\right)^{j} \frac{\Xi_{i, j}\left(\Lambda_{b c_{1}}\right) \lambda_{\langle i\rangle}^{-j} y^{j-1}}{(j-1) !} e^{-\left(y / \lambda_{\langle i\rangle} \mu_{1}\right)},
$$

Let $\mu_{1} \gamma_{b c_{1}}=y$ and $\mu_{2} \gamma_{b c_{2}}=z$; the PDFs of $\mu_{1} \gamma_{b c_{1}}$ and $\mu_{2} \gamma_{b c_{2}}$ can be obtained as

$$
f_{z}(z)=\sum_{i=1}^{\rho\left(\Lambda_{b c_{2}}\right)} \sum_{j=1}^{\delta_{i}\left(\Lambda_{b c_{2}}\right)}\left(\frac{1}{\mu_{2}}\right)^{j} \Xi_{i, j}\left(\Lambda_{b c_{2}}\right) \frac{\lambda_{\langle i\rangle}^{-j} z^{j-1}}{(j-1) !} e^{-\left(z / \lambda_{\langle i\rangle} \mu_{2}\right)} .
$$

By utilizing (23), (B.3), and (B.4), with the similar steps of Appendix A, we can obtain

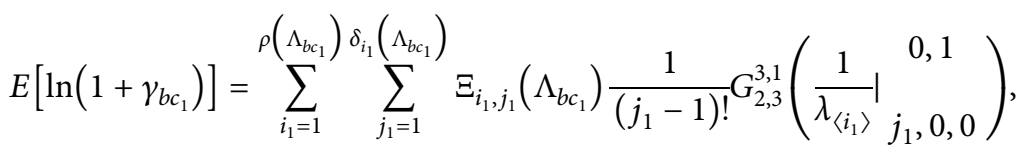

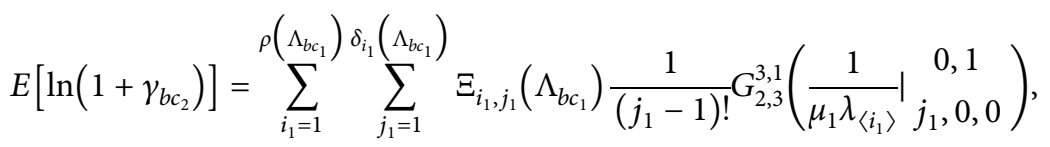

$$
\begin{aligned}
& E\left[\ln \left(1+\mu_{1} \gamma_{b c_{1}}\right)\right]=\sum_{i_{2}=1}^{\rho\left(\Lambda_{b c_{2}}\right)} \sum_{j_{2}=1}^{\delta_{i_{2}}\left(\Lambda_{b c_{2}}\right)} \Xi_{i_{2}, j_{2}}\left(\Lambda_{b c_{2}}\right) \frac{1}{\left(j_{2}-1\right) !} G_{2,3}^{3,1}\left(\frac{1}{\lambda_{\left\langle i_{2}\right\rangle}} \underset{j_{2}, 0,0}{0,1}\right), \\
& E\left[\ln \left(1+\mu_{2} \gamma_{b c_{2}}\right)\right]=\sum_{i_{2}=1}^{\rho\left(\Lambda_{b c_{2}}\right)} \sum_{j_{2}=1}^{\delta_{i_{2}}\left(\Lambda_{b c_{2}}\right)} \Xi_{i_{2}, j_{2}}\left(\Lambda_{b c_{2}}\right) \frac{1}{\left(j_{2}-1\right) !} G_{2,3}^{3,1}\left(\frac{1}{\mu_{2} \lambda_{\left\langle i_{2}\right\rangle} \mid} \underset{j_{2}, 0,0}{0,1}\right) .
\end{aligned}
$$


By substituting (B.5)-(B.8) into (B.1), (37) can be derived. The proof of Theorem 2 is completed.

\section{Data Availability}

No data were used to support this study.

\section{Conflicts of Interest}

The authors declare that they have no conflicts of interest.

\section{Acknowledgments}

This work was supported by the National Science Foundation of China, under Grants 61901502 and 62001517, the National Postdoctoral Program for Innovative Talents, under Grant BX20200101, and the Research Project of NUDT, under Grants ZK18-02-11 and 18-QNCXJ-029 and in part by the Research Project of Space Engineering University, under Grants 2020XXAQ01 and 2019XXAQ05 and Science and Technology Innovation Cultivation Fund of Space Engineering University. Thanks to all the authors for their contribution to this paper.

\section{References}

[1] B. Li, Z. Fei, C. Zhou, and Y. Zhang, "Physical-layer security in space information networks: a survey," IEEE Internet of Things Journal, vol. 7, no. 1, pp. 33-52, 2020.

[2] K. Guo, K. An, B. Zhang et al., "Physical layer security for multiuser satellite communication systems with thresholdbased scheduling scheme," IEEE Transactions on Vehicular Technology, vol. 69, no. 5, pp. 5129-5141, 2020.

[3] K. An, M. Lin, T. Liang et al., "Performance analysis of multiAntenna hybrid satellite-Terrestrial relay networks in the presence of interference," IEEE Transactions on Communications, vol. 63, no. 11, pp. 4390-4404, 2015.

[4] X. Li, Q. Wang, Y. Liu, T. A. Tsiftsis, Z. Ding, and A. Nallanathan, "UAV-aided multi-way NOMA networks with residual hardware impairments," IEEE Wireless Communications Letters, vol. 9, no. 9, pp. 1538-1542, 2020.

[5] H. Kong, M. Lin, W.-P. Zhu, H. Amindavar, and M.-S. Alouini, "Multiuser scheduling for asymmetric FSO/RF links in satellite-UAV-terrestrial networks," IEEE Wireless Communications Letters, vol. 9, no. 8, pp. 1235-1239, 2020.

[6] M. Lin, Z. Lin, W.-P. Zhu, and J.-B. Wang, "Joint beamforming for secure communication in cognitive satellite terrestrial networks," IEEE Journal on Selected Areas in Communications, vol. 36, no. 5, pp. 1017-1029, 2018.

[7] X. Li, Y. Zheng, W. U. Khan et al., "Physical layer security of cognitive ambient backscatter communications for green internet-of-things," IEEE Transactions on Green Communications and Networking, vol. 5, no. 3, pp. 1066-1076, 2021.

[8] X. Yue, Y. Liu, Y. Yao, X. Li, R. Liu, and A. Nallanathan, "Secure communications in a unified non-orthogonal multiple access framework," IEEE Transactions on Wireless Communications, vol. 19, no. 3, pp. 2163-2178, 2020.

[9] K. An, Y. Li, X. Yan, and T. Liang, "On the performance of cache-enabled hybrid satellite-terrestrial relay networks," IEEE Wireless Communications Letters, vol. 8, no. 5, pp. 1506-1509, 2019.

[10] X. Zhang, D. Guo, K. An, G. Zheng, S. Chatzinotas, and B. Zhang, "Auction-based multichannel cooperative spectrum sharing in hybrid satellite-terrestrial IoT networks," IEEE Internet of Things Journal, vol. 8, no. 8, pp. 7009-7023, 2021.

[11] Z. Lin, M. Lin, W.-P. Zhu, J.-B. Wang, and J. Cheng, "Robust secure beamforming for wireless powered cognitive satelliteterrestrial networks," IEEE Transactions on Cognitive Communications and Networking, vol. 7, no. 2, pp. 567-580, 2021.

[12] K. Guo, K. An, B. Zhang et al., "On the performance of the uplink satellite multiterrestrial relay networks with hardware impairments and interference," IEEE Systems Journal, vol. 13, no. 3, pp. 2297-2308, 2019.

[13] K. An and T. Liang, "Hybrid satellite-terrestrial relay networks with adaptive transmission," IEEE Transactions on Vehicular Technology, vol. 68, no. 12, pp. 12448-12452, 2019.

[14] K. Guo, M. Lin, B. Zhang, W.-P. Zhu, J.-B. Wang, and T. A. Tsiftsis, "On the performance of LMS communication with hardware impairments and interference," IEEE Transactions on Communications, vol. 67, no. 2, pp. 1490-1505, 2019.

[15] V. Bankey and P. K. Upadhyay, "Physical layer security of multiuser multirelay hybrid satellite-terrestrial relay networks," IEEE Transactions on Vehicular Technology, vol. 68, no. 3, pp. 2488-2501, 2019.

[16] Q. Huang, M. Lin, W.-P. Zhu, S. Chatzinotas, and M.-S. Alouini, "Performance analysis of integrated satelliteterrestrial multiantenna relay networks with multiuser scheduling," IEEE Transactions on Aerospace and Electronic Systems, vol. 56, no. 4, pp. 2718-2731, 2020.

[17] Y. Qu, H. Dai, H. Wang et al., "Service provisioning for UAVenabled mobile edge computing," IEEE Journal on Selected Areas in Communications, vol. 39, no. 11, pp. 3287-3305, 2021.

[18] X. Liu, Y. Liu, and Y. Chen, "Machine learning empowered trajectory and passive beamforming design in UAV-RIS wireless networks," IEEE Journal on Selected Areas in Communications, vol. 39, no. 7, pp. 2042-2055, 2021.

[19] J. Cui, Y. Liu, and A. Nallanathan, "Multi-agent reinforcement learning-based resource allocation for UAV networks," IEEE Transactions on Wireless Communications, vol. 19, no. 2, pp. 729-743, 2020.

[20] Q. Huang, M. Lin, J.-B. Wang, T. A. Tsiftsis, and J. Wang, "Energy efficient beamforming schemes for satellite-aerialterrestrial networks," IEEE Transactions on Communications, vol. 68, no. 6, pp. 3863-3875, 2020.

[21] Z. Lin, M. Lin, T. de Cola, J.-B. Wang, W.-P. Zhu, and J. Cheng, "Supporting IoT with rate-splitting multiple access in satellite and aerial-integrated networks," IEEE Internet of Things Journal, vol. 8, no. 14, pp. 11123-11134, 2021.

[22] X. Zhang, B. Zhang, K. An, G. Zheng, S. Chatzinotas, and D. Guo, "Stochastic geometry-based analysis of cache-enabled hybrid satellite-aerial-terrestrial networks with non-orthogonal multiple access," IEEE Transactions on Wireless Communications, p. 1, 2021.

[23] X. Liu, C. Gu, K. Guo, M. Cheng, M. Lin, and W.-P. Zhu, "Robust beamforming and outage performance of uplink multiuser satellite-aerial-terrestrial networks with mixed RFFSO channels," IEEE Photonics Journal, vol. 13, no. 4, pp. 1-8, 2021.

[24] Q. Huang, M. Lin, W.-P. Zhu, J. Cheng, and M.-S. Alouini, "Uplink massive access in mixed RF/FSO satellite-aerialterrestrial networks," IEEE Transactions on Communications, vol. 69, no. 4, pp. 2413-2426, 2021.

[25] X. Li, Y. Zheng, M. D. Alshehri et al., "Cognitive AmBCNOMA IoV-MTS networks with IQI: reliability and security 
analysis," IEEE Transactions on Intelligent Transportation Systems, pp. 1-12, 2021.

[26] K. An, M. Lin, W.-P. Zhu, Y. Huang, and G. Zheng, "Outage performance of cognitive hybrid satellite-terrestrial networks with interference constraint," IEEE Transactions on Vehicular Technology, vol. 65, no. 11, pp. 9397-9404, 2016.

[27] Y. Ruan, Y. Li, C.-X. Wang, R. Zhang, and H. Zhang, "Power allocation in cognitive satellite-vehicular networks from energy-spectral efficiency tradeoff perspective," IEEE Transactions on Cognitive Communications and Networking, vol. 5, no. 2, pp. 318-329, 2019.

[28] K. An, J. Ouyang, M. Lin, and T. Liang, "Outage analysis of multi-antenna cognitive hybrid satellite-terrestrial relay networks with beamforming," IEEE Communications Letters, vol. 19, no. 7, pp. 1157-1160, 2015.

[29] P. K. Sharma, P. K. Upadhyay, D. B. da Costa, P. S. Bithas, and A. G. Kanatas, "Performance analysis of overlay spectrum sharing in hybrid satellite-terrestrial systems with secondary network selection," IEEE Transactions on Wireless Communications, vol. 16, no. 10, pp. 6586-6601, 2017.

[30] K. Guo, K. An, B. Zhang, Y. Huang, and G. Zheng, "Outage analysis of cognitive hybrid satellite-terrestrial networks with hardware impairments and multi-primary users," IEEE Wireless Communications Letters, vol. 7, no. 5, pp. 816-819, 2018.

[31] Z. Lin, M. Lin, B. Champagne, W.-P. Zhu, and N. Al-Dhahir, "Secure beamforming for cognitive satellite terrestrial networks with unknown eavesdroppers," IEEE Systems Journal, vol. 15, no. 2, pp. 2186-2189, 2021.

[32] V. Singh, S. Solanki, P. K. Upadhyay, D. B. da Costa, and J. M. Moualeu, "Performance analysis of hardware-impaired overlay cognitive satellite-terrestrial networks with adaptive relaying protocol," IEEE Systems Journal, vol. 15, no. 1, pp. 192-203, 2021.

[33] L. Yang, H. Jiang, Q. Ye et al., "Opportunistic adaptive nonorthogonal multiple access in multiuser wireless systems: probabilistic user scheduling and performance analysis," IEEE Transactions on Wireless Communications, vol. 19, no. 9, pp. 6065-6082, 2020.

[34] L. Yang, H. Jiang, Q. Ye et al., "On the application of cooperative NOMA to spatially random wireless caching networks," IEEE Transactions on Vehicular Technology, vol. 70, no. 11, pp. 12055-12071, 2021.

[35] M. Jia, Q. Gao, Q. Guo, X. Gu, and X. Shen, "Power multiplexing NOMA and bandwidth compression for satelliteterrestrial networks," IEEE Transactions on Vehicular Technology, vol. 68, no. 11, pp. 11107-11117, 2019.

[36] Z. Lin, M. Lin, J.-B. Wang, T. de Cola, and J. Wang, "Joint beamforming and power allocation for satellite-terrestrial integrated networks with non-orthogonal multiple access," IEEE Journal of Selected Topics in Signal Processing, vol. 13, no. 3, pp. 657-670, 2019.

[37] X. Yan, H. Xiao, K. An, G. Zheng, and S. Chatzinotas, "Ergodic capacity of NOMA-based uplink satellite networks with randomly deployed users," IEEE Systems Journal, vol. 14, no. 3, pp. 3343-3350, 2020.

[38] K. Guo, K. An, F. Zhou, T. A. Tsiftsis, G. Zheng, and S. Chatzinotas, "On the secrecy performance of NOMA-based integrated satellite multiple-terrestrial relay networks with hardware impairments," IEEE Transactions on Vehicular Technology, vol. 70, no. 4, pp. 3661-3676, 2021.

[39] R. Liu, K. Guo, K. An, S. Zhu, and H. Shuai, "NOMA-based integrated satellite-terrestrial relay networks under spectrum sharing environment," IEEE Wireless Communications Letters, vol. 10, no. 6, pp. 1266-1270, 2021.

[40] R. Liu, K. Guo, K. An, and S. Zhu, "NOMA-based overlay cognitive integrated satellite-terrestrial relay networks with secondary network selection," IEEE Transactions on Vehicular Technology, p. 1, 2021.

[41] I. S. Gradshteyn and I. M. Ryzhik, Table of Intergals, Series, and Products, Academic Press, Amsterdam, Netherlands, 7th edition, 2007.

[42] N. I. Miridakis, D. D. Vergados, and A. Michalas, "Dual-hop communication over a satellite relay and Shadowed Rician channels," IEEE Transactions on Vehicular Technology, vol. 64, no. 9, pp. 4031-4040, 2015.

[43] "The wolfram function site," https://functions.wolfram.com.

[44] A. Bletsas, H. Shin, and M. Win, "Cooperative communications with outage-optimal opportunistic relaying," IEEE Transactions on Wireless Communications, vol. 6, no. 9, pp. 3450-3460, 2007.

[45] G. Farhadi and N. C. Beaulieu, "On the ergodic capacity of multi-hop wireless relaying systems," IEEE Transactions on Wireless Communications, vol. 8, no. 5, pp. 2286-2291, 2009.

[46] A. P. Prudnikov, Y. A. Brychkov, and O. I. Marichev, Integralsand Series, Volume 3: More Special Functions, ser. Gordon and Breach, New York, NY, USA, 1990. 